\title{
TITLE:
}

\section{Turbulence Effect on Cloud Radiation}

\author{
$\operatorname{AUTHOR}(\mathrm{S})$ :
}

Matsuda, K.; Onishi, R.; Kurose, R.; Komori, S.

\section{CITATION:}

Matsuda, K....[et al]. Turbulence Effect on Cloud Radiation. Physical Review Letters 2012, 108(22): 224502.

ISSUE DATE:

2012-05-30

URL:

http://hdl.handle.net/2433/188012

RIGHT:

(C2012 American Physical Society 


\title{
Turbulence Effect on Cloud Radiation
}

\author{
K. Matsuda, ${ }^{1}$ R. Onishi, ${ }^{2}$ R. Kurose,${ }^{1}$ and S. Komori ${ }^{1}$ \\ ${ }^{1}$ Department of Mechanical Engineering and Science, Advanced Research Institute of Fluid Science and Engineering, \\ Kyoto University, Kyoto 606-8501, Japan \\ ${ }^{2}$ Earth Simulator Center, Japan Agency for Marine-Earth Science and Technology (JAMSTEC), Yokohama 236-0001, Japan
}

(Received 5 July 2011; published 30 May 2012)

\begin{abstract}
The effect of turbulent clustering of water droplets on radiative transfer is investigated by means of both a three-dimensional direct numerical simulation of particle-laden homogeneous isotropic turbulence and a radiative transfer simulation based on a Monte Carlo photon tracing method. The results show that turbulent clustering causes the formation of void regions of droplets and hence increases the direct transmittance. This effect decreases as the turbulent Reynolds number increases and is estimated to be negligibly small under the conditions in real clouds.
\end{abstract}

Clouds play an important role for the radiative heat budget on Earth. For reliable predictions of climate change, cloud radiation must be evaluated accurately. However, we still have many uncertainties about cloud radiation processes. For example, the effect of turbulence on cloud radiation has not been well understood.

Cloud turbulence forms an inhomogeneous distribution of water droplets, often referred to as turbulent clustering or preferential concentration [1-3]. Mishchenko [4] investigated the effect of centimeter-scale inhomogeneity of spatial distribution of cloud droplets in a 10-meter-scale cloud volume. His probabilistic theory estimated that the small-scale inhomogeneity does not affect radiative scattering. This suggests that the radiative transfer process follows the conventional exponential extinction law, which is called the Beer-Lambert law. The effect on extinction was investigated by Kostinski $[5,6]$. He showed that the radiative transfer process does not follow the BeerLambert law in a medium with spatially correlated particles. Borovoi [7] further showed that the Beer-Lambert law is applicable only to the medium with small-scale inhomogeneity, but not to the one with large-scale inhomogeneity. All their conclusions were, however, based on estimates of the particle distributions with specific spatial correlations without detailed discussions. Therefore, the effect of inhomogeneity due to turbulence on radiative transfer is still unclear.

This study, therefore, aims to investigate the effect of inhomogeneity on radiative properties based on the particle distributions obtained from physics-based simulations. Direct numerical simulations (DNS) of three-dimensional particle-laden homogeneous isotropic turbulence were performed to obtain realistic spatial distributions of droplets in turbulence. Then, the radiative properties of the obtained media with clustering droplets were calculated based on a Monte Carlo photon tracing method.

The governing equations of air turbulence in the DNS are the continuity and incompressible Navier-Stokes equations. The fourth-order central finite-difference was used for advection [8] and viscous terms. The second-order Runge-Kutta method was used for time evolution. Steady turbulence was formed by applying the external force which keeps the intensity of a large-scale eddy. The reduced-communication forcing [9], which can suppress the computational cost arising from the domain decomposition for parallel computing, was used for the external forcing.

The droplet motions were calculated by the Lagrangian tracking method $[10,11]$. The governing equation of the motion is $\frac{d v_{i}}{d t}=-\left(v_{i}-u_{i}\right) / T_{p}$, where $u_{i}$ is the fluid velocity, $v_{i}$ the droplet velocity, and $T_{p}$ the droplet relaxation time. Droplets were assumed as Stokes particles, whose relaxation time $T_{p}$ is given as $T_{p}=\left(\rho_{p} / \rho_{g}\right)\left(2 r^{2} / 9 \nu\right)$, where $r$ is the droplet radius, $\rho_{p}$ the water density, $\rho_{g}$ the air density, and $\nu$ the kinematic viscosity.

Isotropic air turbulence and droplet motions were simulated in a cubic domain of length $2 \pi L_{0}$, where $L_{0}$ is the representative length scale. Periodic boundary conditions were applied in all three directions.

This study performed two air flow simulations. Table I lists the computational conditions and flow properties including the rms value of velocity fluctuation $u^{\prime}$, the Kolmogorov length scale $l_{\eta}$, and the turbulent Reynolds number based on the Taylor microscale $\operatorname{Re}_{\lambda}$. The numbers of grid points were set to $64^{3}$ in a low Reynolds number case or $1000^{3}$ in a high Reynolds number case. In both cases, the droplets were distributed randomly in the domain after the turbulence was fully developed. The droplet radius was set to $20 \mu \mathrm{m}$ so that the Stokes number $\mathrm{St}$

TABLE I. Numerical conditions and flow properties.

\begin{tabular}{lcccc}
\hline \hline & $L_{0}[\mathrm{~m}]$ & $u^{\prime}[\mathrm{m} / \mathrm{s}]$ & $l_{\eta}\left[10^{-4} \mathrm{~m}\right]$ & $\operatorname{Re}_{\lambda}[-]$ \\
\hline Low $\operatorname{Re}_{\lambda}$ & 0.0050 & 0.20 & 2.69 & 54.3 \\
$\mathrm{High} \mathrm{Re}_{\lambda}$ & 0.0659 & 0.50 & 2.73 & 340 \\
\hline \hline
\end{tabular}


(= $T_{p} / T_{\eta}$, where $T_{\eta}$ is the Kolmogorov time scale) was close to unity. St of cloud droplets typically ranges from $10^{-3}$ to $10^{-1}$ [12], but St of large droplets reaches as much as 10. Since turbulent clustering becomes the most pronounced when $\mathrm{St} \sim 1$, this study employs $\mathrm{St}=1$ to evaluate the maximum effect of turbulent clustering. The maximum number of droplets was $50 \times 10^{6}$ in the high Reynolds number case. In the DNS, collisions between droplets were neglected to reduce the computational cost. In order to avoid the reduction of the volume fraction of droplets due to their overlapping, the overlapping droplets were shifted so that the minimum distance between the droplets is $2 r$.

The radiative transfer properties for the media with clustering droplets were obtained by using a Monte Carlo photon tracing method, where transports of a number of photons were traced stochastically. In the conventional Monte Carlo photon tracing method [13], a free path length of a photon is determined stochastically by the BeerLambert law. On the other hand, in our Monte Carlo photon tracing method, geometrical collisions between photons and individual droplets were taken into account. A photon was assumed to collide with a droplet closest to the photon among droplets which satisfy the following criteria: $\left|\left(\mathbf{x}_{p}-\mathbf{x}_{0}\right) \times \hat{\mathbf{k}}\right|<r_{\text {ext }}$ and $\left(\mathbf{x}_{p}-\mathbf{x}_{0}\right) \cdot \hat{\mathbf{k}}>0$. Here, $\mathbf{x}_{0}$ and $\mathbf{x}_{p}$ are the positions of the photon and droplet. $\hat{\mathbf{k}}$ is the unit traveling direction vector of photon. $r_{\mathrm{ext}}$ is the droplet extinction radius, which we defined as $r_{\mathrm{ext}}=$ $\sqrt{\sigma_{\text {ext }} / \pi}$, where $\sigma_{\text {ext }}$ is the extinction cross section.

Absorption by droplets was taken into account by using a weight $w^{(m)}$ proportional to the radiative power of each photon, where the superscript $(m)$ indicates the order of collision. On a collision of order $m+1$, the weight was scaled as $w^{(m+1)}=\omega w^{(m)}$, and the photon was scattered with the weight $w^{(m+1)}$. Here, $\omega$ is the single scattering albedo of the droplet, which is defined as the ratio of the scattering cross section $\sigma_{\text {scat }}$ to the extinction cross section $\sigma_{\text {ext }}$. The direction of the scattered photon was determined by the scattering angle $\theta$ and azimuth angle $\phi$. $\theta$ was chosen based on the scattering phase function $p(\theta)$, whereas $\phi$ was chosen randomly.

Photons were radiated downward ( $-y$ direction) from random positions on the top boundary of the computational domain. Periodic boundary conditions for photon movement were applied in $x$ and $z$ directions. The photons were traced until they left the domain from the top or bottom boundary, or their weights $w^{(m)}$ became smaller than $10^{-9}$ due to the absorption.

In this study, radiative transfer was calculated for the wavelengths of $\lambda=0.5 \mu \mathrm{m}$ and $3.0 \mu \mathrm{m}$. The former is a visible radiation, which is not absorbed by droplets, whereas the latter is an infrared radiation, which is partially absorbed by droplets. The extinction cross section $\sigma_{\text {ext }}$, single scattering albedo $\omega$, and scattering phase function $p(\theta)$ for these wavelengths were obtained from the Mie scattering theory [14]. The values of $\sigma_{\text {ext }}$ and $\omega$ for a water droplet of $r=20 \mu \mathrm{m}$ are $2.03 \pi r^{2}$ and 1.00 for the wavelength of $\lambda=0.5 \mu \mathrm{m}$, whereas $2.15 \pi r^{2}$ and 0.53 for the wavelength of $\lambda=3.0 \mu \mathrm{m}$. Absorption by gases was ignored for simplicity since the volume absorption coefficient of gases was much smaller than that of droplet medium in most of the cases. For reference, radiative transfer was also simulated for random dispersion of droplets.

Figure 1 shows the randomly dispersed droplets, and the clusterings for the low and high Reynolds number turbulence. The domain size and number density of the distribution of randomly dispersed droplets are the same as the low Reynolds number case. Turbulent clustering is clearly observed in the distributions obtained from DNS, whereas it is not observed in the random case. In order to compare quantitatively the scales of clusters, the pair correlation function (PCF) $h(l)$ of the clustering droplets was calculated. The PCF corresponds to the spatial correlation of number density fluctuation at the separation $l$, and is defined as $h(l)=\left\langle\left[n_{p, \text { local }}(x)-n_{p}\right]\left[n_{p, \text { local }}(x+l)-\right.\right.$ $\left.\left.n_{p}\right]\right\rangle / n_{p}^{2}$, where $n_{p}$ is the mean droplet number density, $n_{p, \text { local }}(x)$ is the local number density, and \langle\rangle indicates the ensemble average. Figure 2 shows the PCF of the spatial
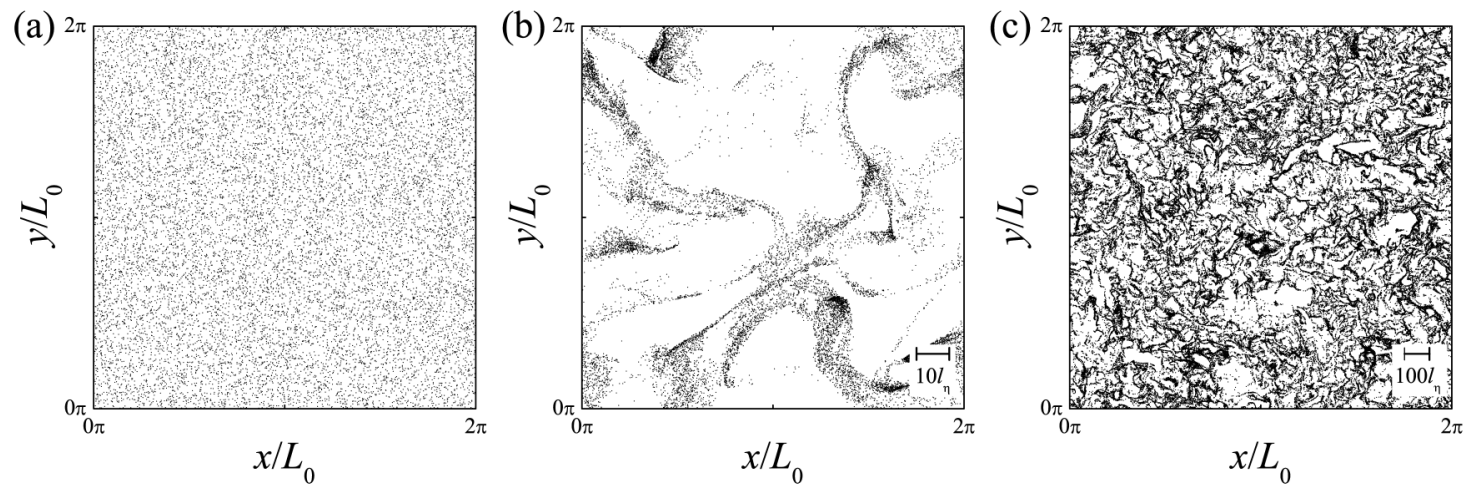

FIG. 1. Droplet distributions for (a) the random case, (b) $\operatorname{Re}_{\lambda}=54.3$, and (c) $\operatorname{Re}_{\lambda}=340$. Droplets within the range of $0<z<4 l_{\eta}$ are drawn. 


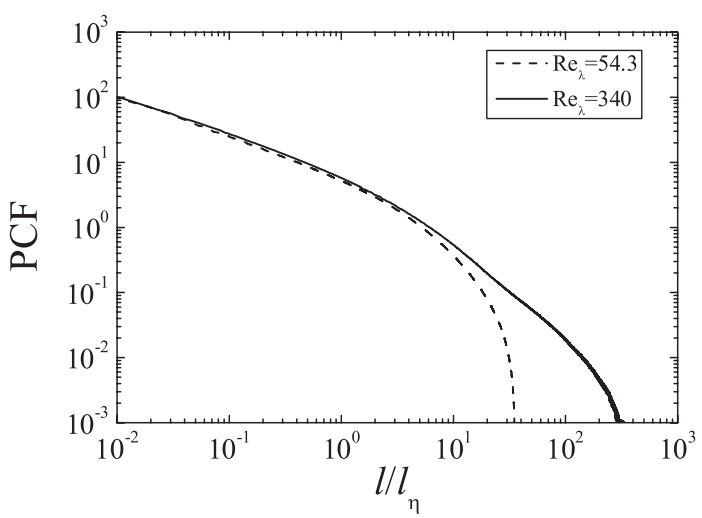

FIG. 2. Pair correlation function (PCF) of turbulent clustering droplets.

distributions for the low and high Reynolds number cases. The PCF of randomly dispersed droplets is 0 in the whole range of $l / l_{\eta}$. It is observed that the PCFs for the both cases are large at $l / l_{\eta}<10$, which indicates that the turbulent clustering are significant in the small scale. The two PCFs are identical in this scale. On the other hand, in the larger scale, the PCFs for the high and low Reynolds number cases are different from each other. This indicates that the droplet distributions are inhomogeneous even in the inertial range, and there is a difference in the inhomogeneity between the high and low Reynolds number cases.

The radiative properties, which include the transmittance $\mathcal{T}$, reflectance $\mathcal{R}$, and absorptance by droplets $\mathcal{A}_{p}$ were evaluated in the radiative transfer simulations. These radiative properties satisfy $\mathcal{T}+\mathcal{R}+\mathcal{A}_{p}=1$. Basically, the radiative properties of a medium with randomly dispersed droplets are determined by the wavelength $\lambda$ and the optical depth $\tau$, which is given by $\tau=\sigma_{\text {ext }} n_{p} \Delta y$, where $\Delta y$ is the depth of the cloud. In this simulation, $\Delta y$ is equal to the depth of computational domain, that is $\Delta y=2 \pi L_{0}$.

Table II shows the transmittance $\mathcal{T}$, reflectance $\mathcal{R}$, and absorptance $\mathcal{A}_{p}$ for visible radiation $(\lambda=0.5 \mu \mathrm{m})$ at the optical depth of $\tau=0.744$ and those for infrared radiation $(\lambda=3.0 \mu \mathrm{m})$ at $\tau=0.785$. As mentioned before, visible radiation is not absorbed by droplets. In the case of visible radiation, $\mathcal{T}$ and $\mathcal{R}$ of the clustering droplets are almost

TABLE II. Radiative properties of the media with clustering droplets and with randomly dispersed droplets for visible radiation $(\lambda=0.5 \mu \mathrm{m})$ and infrared radiation $(\lambda=3.0 \mu \mathrm{m})$.

\begin{tabular}{ccccc}
\hline \hline & & $\mathcal{T}$ & $\mathcal{R}$ & $\mathcal{A}_{\mathrm{p}}$ \\
\hline$\lambda=0.5 \mu \mathrm{m}$ & $\operatorname{Random~case~}$ & 0.973 & $2.66 \times 10^{-2}$ & 0.00 \\
& $\operatorname{Re}_{\lambda}=54.3$ & 0.973 & $2.65 \times 10^{-2}$ & 0.00 \\
& $\operatorname{Re}_{\lambda}=340$ & 0.973 & $2.65 \times 10^{-2}$ & 0.00 \\
$\lambda=3.0 \mu \mathrm{m}$ & $\operatorname{Random~case~}$ & 0.684 & $3.85 \times 10^{-3}$ & 0.312 \\
& $\operatorname{Re}_{\lambda}=54.3$ & 0.699 & $3.53 \times 10^{-3}$ & 0.297 \\
& $\operatorname{Re}_{\lambda}=340$ & 0.686 & $3.81 \times 10^{-3}$ & 0.311 \\
\hline \hline
\end{tabular}

the same as those of the randomly dispersed droplets for both low and high Reynolds number cases. On the other hand, in the case of infrared radiation, $\mathcal{T}$ of the clustering droplets is larger than that of the randomly dispersed droplets, and $\mathcal{R}$ of the clustering droplets is smaller than that of the randomly dispersed droplets. In addition, $\mathcal{A}_{p}$ of the clustering droplets is smaller than that of the randomly dispersed droplets. These differences are smaller in the high Reynolds number case than in the low Reynolds number case.

The differences are attributed to the change in the relation between the transmission and scattering. The transmittance $\mathcal{T}$ consists of diffuse transmittance $\mathcal{T}_{\text {diffuse }}$ and direct transmittance $\mathcal{T}_{\text {direct }}$. $\mathcal{T}_{\text {diffuse }}$ and $\mathcal{T}_{\text {direct }}$ are the portions of the radiation passing through the medium with and without being scattered, respectively. Figure 3 shows the direct transmittance $\mathcal{T}_{\text {direct }}$ of infrared radiation of the media with clustering droplets for several values of optical depth $\tau$, which were set by changing the droplet number density $n_{p}$. The solid line indicates the direct transmittance $\left(\mathcal{T}_{\text {direct }}\right)_{\text {random }}$ for randomly dispersed droplets. It is given theoretically by the Beer-Lambert law, which is expressed as $\left(\mathcal{T}_{\text {direct }}\right)_{\text {random }}=\exp (-\tau)$. $\mathcal{T}_{\text {direct }}$ in the low Reynolds number case is found to be larger than $\left(\mathcal{T}_{\text {direct }}\right)_{\text {random }}$ obtained from the Beer-Lambert law. This result indicates that the clustering increases the number of photons which pass through without being scattered. This is due to the void regions in the clustering distribution, as observed in Fig. 1. The increase of the photons transmitting without being scattered enhances $\mathcal{T}$, and the decrease of the photons colliding with droplets suppresses $\mathcal{R}$ and $\mathcal{A}_{p}$. On the other hand, $\mathcal{T}_{\text {direct }}$ in the high Reynolds number case almost corresponds to the Beer-Lambert law. The difference between the high and low Reynolds number cases is due to the difference in relative scale of cluster to the domain. Since $\tau$ is determined by the depth of domain $2 \pi L_{0}$, the mean free paths of photons relative to the domain size are comparable with each other in the media

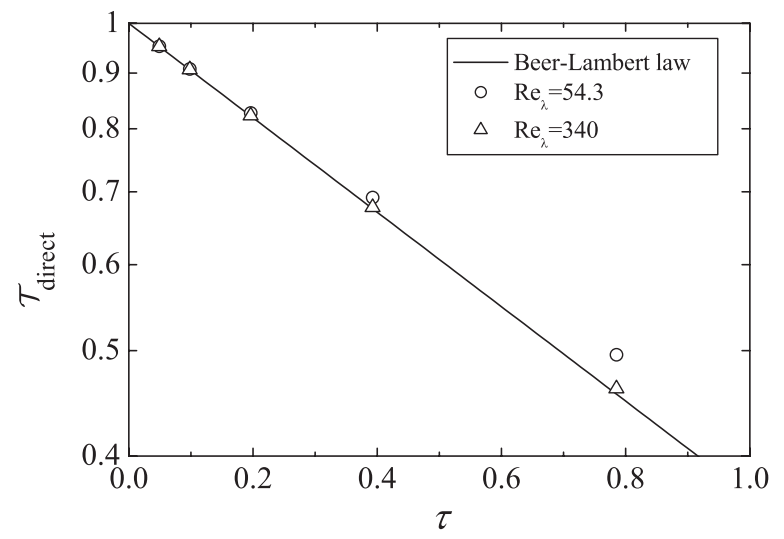

FIG. 3. Comparison of direct transmittance of the media with clustering droplets and that obtained from the Beer-Lambert law for infrared radiation. 


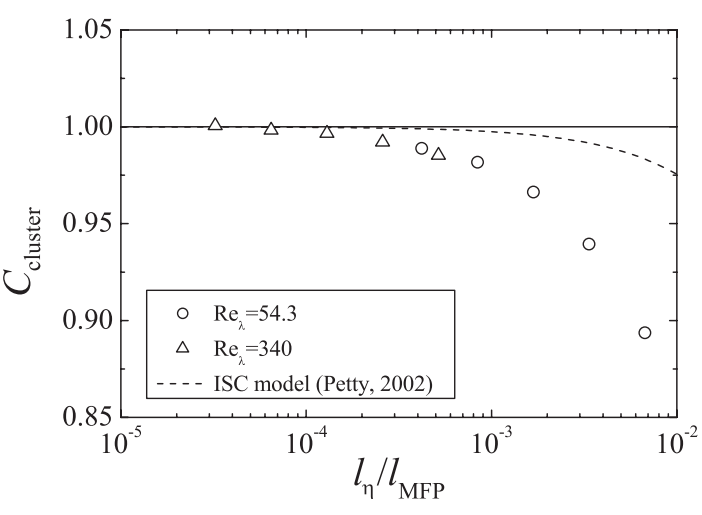

FIG. 4. Correction coefficient for direct transmittance of the media with clustering droplets for infrared radiation.

with the same $\tau$. Therefore, the cluster scale relative to the mean free path (MFP) of photons decreases as $\mathrm{Re}_{\lambda}$ increases. This is why the effect of clustering on radiative transfer weakens as $\operatorname{Re}_{\lambda}$ increases.

In order to discuss the effect of turbulent clustering on real clouds, it is necessary to consider the scale difference between our simulation and real clouds. The geometric depth of real clouds ranges from $10^{2}$ to $10^{4} \mathrm{~m}[15,16]$, which is much larger than the domain size of our simulations. Furthermore, it is not feasible to perform the DNS in the domain size comparable to real clouds. Therefore, it is required to develop a model to estimate the effect of clustering on cloud radiation. In this study, we modified the Beer-Lambert law by defining a correction coefficient $C_{\text {cluster }}$ as $\left(\mathcal{T}_{\text {direct }}\right)_{\text {cluster }}=\exp \left(-C_{\text {cluster }} \tau\right)$. $C_{\text {cluster }}$ corresponds to the ratio of effective optical depth to the optical depth $\tau$ in the random case, which was intensely discussed by, e.g., Davis et al. [17], Cairns et al. [18], and Petty [19]. For example, Petty [19] proposed the independently scattering cloudlet (ISC) model. The ISC model treats an inhomogeneous cloud distribution as a random ensemble of independently scattering discrete cloudlets, which are modeled as homogeneous spheres. According to the ISC model, $C_{\text {cluster }}$ is parametrized by the optical diameter $\tau^{\prime}$ of the cloudlet, which is defined as the ratio of cloudlet diameter to the mean free path of photons in the cloudlet. Figure 4 shows the correction coefficient $C_{\text {cluster }}$ versus the ratio of the Kolmogorov length scale $l_{\eta}$ to the mean free path of photons in a medium with randomly dispersed droplets, $l_{\mathrm{MFP}}=\left(\sigma_{\mathrm{ext}} n_{p}\right)^{-1}$. $C_{\text {cluster }}$ obtained from the low and high Reynolds number cases are plotted with that obtained from the ISC model by assuming $\tau^{\prime}=\left(n_{p, \text { cluster }} / n_{\mathrm{p}}\right)\left(l_{\eta} / l_{\mathrm{MFP}}\right) \approx\left[h\left(l_{\eta}\right)+1\right]\left(l_{\eta} / l_{\mathrm{MFP}}\right)$, where $n_{p \text {,cluster }}$ is the local droplet number density in clusters. $C_{\text {cluster }}$ obtained from our simulations are observed to be smaller than unity, which corresponds to the increase of $\mathcal{T}_{\text {direct }}$ in Fig. 3. The value of $C_{\text {cluster }}$ estimated by the ISC model is much closer to unity than that from our simulations. Thus, the ISC model does not represent the effect of turbulent clustering on radiative transfer. In real clouds, the mean free path $l_{\mathrm{MFP}}$ is in the range from $10^{-3}$ to $10^{0} \mathrm{~m}$ since the number density $n_{p}$ is within the range from $10^{7}$ to $10^{9} \mathrm{~m}^{-3}$ [20], and the typical droplet radius is $10 \mu \mathrm{m}$ [20]. On the other hand, the typical Kolmogorov length scale $l_{\eta}$ is $1 \mathrm{~mm}$ [12]. Therefore, $l_{\eta} / l_{\mathrm{MFP}}$ in real clouds is in the range from $10^{-6}$ to $10^{-3}$. In this range of $l_{\eta} / l_{\mathrm{MFP}}$, the correction coefficient $C_{\text {cluster }}$ for $\mathrm{St}=1$ is larger than 0.98 . Therefore, the effect of turbulent clustering on cloud radiation is estimated to be negligibly small as anticipated by Mishchenko [4].

This study employed monodispersed spherical water droplets with $\mathrm{St}=1$. As the clustering being the most pronounced for $S t \sim 1$, the effect of clustering is smaller in real clouds, which contain droplets with various values of the Stokes number. It should be noted that clouds often contain ice particles. Ice particles usually have complex geometry, which adds randomness to particle motions. The additive randomness decreases the effect of turbulent clustering. Thus, this study has discussed the maximum possible effect of turbulent clustering in clouds by performing the physics-based simulations and revealed that the effect is negligibly small.

This research was supported by Grant-in-Aid for Young Scientists (A) (No. 20686015) and by Grant-in-Aid for JSPS Fellows (No. 21.241).

[1] M. Maxey, J. Fluid Mech. 174, 441 (1987).

[2] K. Squires and J. Eaton, Phys. Fluids A 3, 1169 (1991).

[3] L. Chen, S. Goto, and J. Vassilicos, J. Fluid Mech. 553, 143 (2006).

[4] M. Mishchenko, Geophys. Res. Lett. 33, L14820 (2006).

[5] A. Kostinski, J. Opt. Soc. Am. A 18, 1929 (2001).

[6] A. Kostinski, J. Opt. Soc. Am. A 19, 2521 (2002).

[7] A. Borovoi, J. Opt. Soc. Am. A 19, 2517 (2002).

[8] Y. Morinishi, T. Lund, O. Vasilyev, and P. Moin, J. Comput. Phys. 143, 90 (1998).

[9] R. Onishi, Y. Baba, and K. Takahashi, J. Comput. Phys. 230, 4088 (2011).

[10] R. Onishi, K. Takahashi, and S. Komori, Phys. Fluids 21, 125108 (2009).

[11] R. Onishi, K. Matsuda, K. Takahashi, R. Kurose, and S. Komori, Int. J. Multiphase Flow 37, 125 (2011).

[12] R. Shaw, Annu. Rev. Fluid Mech. 35, 183 (2003).

[13] A. Macke, D. Mitchell, and L. von Bremen, Phys. Chem. Earth B 24, 237 (1999).

[14] C. Bohren and D. Huffman, Absorption and Scattering of Light by Small Particles (Wiley, New York, 1983).

[15] J. London, J. Meteorol. 9, 145 (1952).

[16] K. Liou, J. Atmos. Sci. 33, 798 (1976).

[17] A. Davis, P. Gabriel, S. Lovejoy, D. Schertzer, and G. Austin, J. Geophys. Res. 95, 11729 (1990).

[18] B. Cairns, A. Lacis, and B. Carlson, J. Atmos. Sci. 57, 700 (2000).

[19] G. Petty, J. Atmos. Sci. 59, 2910 (2002).

[20] A. Kokhanovsky, Earth-Sci. Rev. 64, 189 (2004). 